\title{
Differential inequalities and the Okamura function.
}

\author{
S. R. BeRNFEld - V. LaKshMHKanthaM (Kingston, R. I.) (*)
}

Summary. - The theroy of differential inequalities and Liapunov functions are used to characterize the integral stability, integral boundedness and integral extendability of solution of differential equations. The Liapunov functions are modifications of the Okamura function.

\section{1. - Introduction.}

The application of Liapunov functions in the theory of perturbed differential equations has been extensive. Recently results on the preservation of stability and boundedness under integrable perturbations have been obtained. In particular CHOW and YoRKE [2] have extended the work of VRKOC [6] concerning integral stability. By using a modification of a function essentially due to OKaMURA [5] and later applied by YoshizaWA [7], they have obtained simpler proofs of Vrkoc's results by characterizing integral stability in terms of the Okamura function. In another paper CHow and one of the authors [1] have extended the notion of integral stability to that of integral boundedness and extendability and have characterized these in terms of an Okamura type function.

The purpose of this paper is to generalize the results in [1] and [2] by using the comparison principle for differential inequalities. In particular we discuss the behavior of solutions of the perturbed differential equation in terms of the Liapunov function and the solutions of the scalar differential equation. This allows us, for example, to deduce the integral stability and boundedness (integral asymptotic stability) without the stronger requirement that the derivative of the Liapunov function be nonpositive (negative definite) which sometimes may be difficult to achieve. Moreover for each of the cases of integral stability, integral asymptotic stability, and integral boundedness we are able to produce a family of Liapunov functions, thus allowing for more flexibility in applications.

Finally we develop the analogue for ultimate boundedness under integrable perturbations. Sufficient conditions for integral ultimate boundedness are given in terms of scalar differential inequalities, where the solutions of the scalar equation are uniformly ultimately bounded. Results of this type for the case of integral stability and integral asymptotic stability have been developed by LAKsHMTKANTHAM and LEELA [4] using the theory of differential inequalities.

(*) Entrata in Redazione il giorno 8 marzo 1972. 
For an extensive treatment of Liapunov theory and differential inequalities the reader is referred to [4].

\section{2. - Notation and preliminaries.}

Let $R^{d}$ denote Euclidean $d$-space and $|\cdot|$ will denote any $d$-dimensional norm. Define $S_{\dot{e}}=\left\{x \in R^{\alpha}:|x|<\varrho\{\right.$. Represent a solution of the unperturbed differential equation

$$
\dot{x}=f(t, x),
$$

through $\left(t_{0}, x_{0}\right)$ with $t_{0} \geqslant 0$ by $n\left(t, t_{0}, x_{0}\right)$. Consider the perturbed differential equation

$$
\dot{x}=f(t, x)+g(t)
$$

and denote a solution of $(\mathrm{P})$ through $\left(t_{0}, x_{0}\right)$ by $x_{p}\left(t, t_{0}, x_{0}\right)$.

Assume that $f(\cdot, x)$ is measurable for each $x, f(t, \cdot)$ is continuous for each $t,|f|$ is bounded on compact subsets of $[0, \infty) \times R^{a}$ and $g:[0, \infty) \rightarrow R^{a}$ is measurable.

Let $V:[0, \infty) \times R^{d} \rightarrow R$ be a Liapunov function. Define the time derivative of $V$ along solutions of (E) as

$$
\dot{V}_{E}(t, x)=\lim _{h \rightarrow 0^{+}} \sup \frac{1}{h}(V(t+h, x(t+h))-V(t, x)) .
$$

If $V$ is Lipschitzean then it is well known [7] that

$$
\dot{V}_{E}(t, x)=\lim _{h \rightarrow 0^{+}} \sup \frac{1}{h} V(t+h, x+h f(t, x))-V(t, x) .
$$

Throughout this paper our perturbation term $g(t)$ will be independent of $x$. As pointed out by HALANAY [3] for integral stability and in [1] for integral boundedness it is no more general to consider perturbation terms $g(t, x)$. Hence since no new results are obtained for the seemingly more general perturbation term $g(t, x)$ we need only consider the perturbation term to depend on $t$. The following definitions will be used.

Definition 1. - The solution $x(t) \equiv 0$ is integrally stable for (E) if for each $\varepsilon>0$, there exists $\delta=\delta(\varepsilon)>0$ and $\eta=\eta(\varepsilon)>0$ such that whenever $\left|\infty_{0}\right|<\delta$ and

$$
\int_{t_{0}}^{\infty}|g(t)| d t<\eta
$$

then $\left|x_{p}\left(t, t_{0}, x_{0}\right)\right|<\varepsilon$ for $t \geqslant t_{0} \geqslant 0$. 
DEFinimion 2. - The solution $x(t) \equiv 0$ is integrally asymptotically stable for (E) if it is integrally stable for $(\mathrm{E})$ and if there exists $\delta_{0}>0$, such that for each $\varepsilon>0$, there exist $T=T(\varepsilon)>0$ and $\eta=\eta(\varepsilon)>0$ such that whenever $\left|x_{0}\right|<\delta_{0}$ and

$$
\int_{t_{0}}^{\infty}|g(t)| d t<\eta
$$

then $\left|x_{p}\left(t, t_{0}, x_{0}\right)\right|<\varepsilon$ for all $t \geqslant t_{0}+T$.

DEFINTtoN 3. - Solutions of (E) are integrally bounded if for each $\alpha>0$ and $\eta>0$ there exists $\beta=\beta(\alpha, \eta)>0$ such that whenever $\left|x_{0}\right|<\alpha$ and

$$
\int_{t_{0}}^{\infty}|g(t)| d t<\eta
$$

then $\left|x_{P}\left(t, t_{0}, x_{0}\right)\right|<\beta$ for $t \geqslant t_{0} \geqslant 0$

DEFINITION 4. - Solutions of (E) are integrally ultimately bounded if for each $\alpha>0$ and $\eta>0$ there exists $\beta=\beta(\eta)$ and $T=T(\alpha)$ such whenever $\left|x_{0}\right|<\alpha$ and

$$
\int_{i_{0}}^{\infty}|g(t)| d t<\eta
$$

then $x_{p}\left(t, t_{0}, x_{0}\right)<\beta$ for $t \geqslant t_{0}+T$.

DEEINITION 5. - Solutions of (E) are integrally extendable if for each $t_{0} \geqslant 0, \alpha>0$, $T>0$ and $\eta>0$ there exists $\beta=\beta\left(t_{0}, T, \alpha, \eta\right)$ such that whenever $x_{0} \mid<\alpha$ and

$$
\int_{t_{0}}^{\infty}|g(t)| d t<\eta
$$

then $\left|x_{p}\left(t, t_{0}, x_{0}\right)\right|<\beta$ for $t \in\left[t_{0}, t_{0}+T\right]$.

Definitions 1 and 2 may be found in [2] while Definitions 3 and 5 appear in [1]. Definition 4 appears to be new.

We shall be comparing the solutions of (E) with the solution $u\left(t, t_{0}, u_{0}\right)$ of the scalar equation

$$
\dot{u}=h(t, u)
$$

where $h:[0, \infty) \times[0, \infty) \rightarrow R$ is continuous. We shall always assume solutions of (S) are uniquely defined on $[0, \infty)$. 
The following properties of the Liapunov functions $V:[0, \infty) \times R^{d} \rightarrow R$ will be used:

$$
|V(t, x)-V(t, y)| \leqslant K|x-y|
$$

for some $K>0$ and for all $x \in R^{d}, y \in R^{d}$, and

$$
\dot{V}_{E}(t, x) \leqslant h(t, V(t, x)) \text {. }
$$

\section{3. - Results.}

We now present our main results with the proofs following in Section 4 .

The following function was first defined by OKamura [5] and used to show the uniqueness of solutions: Define

$$
\Gamma^{*}(t, x)= \begin{cases}\inf _{p \in \Delta(t, x)} \int_{0}^{t} \mid \dot{\varphi}(s)-f(s, \varphi(s)(\mid d s, & t>0 \\ |x|, & t=0\end{cases}
$$

where $A(t, x)$ is the set of absolutely continuous function $\{\varphi(\cdot)\}$ defined on $[0, t]$ satisfying $\varphi(0)=0$ and $\varphi(t)=x$. YosmzaWA [7] further developed the function, and recently CHOW and YORKE [2] used this function to characterize integral stability while CHow and one of the authors [1] utilized the Okamura function in their study of integral boundedness and extendability.

Here, we shall make use of the more general function

$$
W_{\psi}(t, x)=u\left(t, 0, V_{\psi}(t, x)\right)
$$

where $u$ is a solution of (S) and

$$
V_{\psi}(t, x)= \begin{cases}\inf _{q \in \mathbb{A}(t, x)} \int_{0}^{t} \psi(s)|\dot{\varphi}(s)-f(s, \varphi(s))| d s, & t>0, \\ |x|, & t=0\end{cases}
$$

where $\psi(t)$ is a positive nondecreasing continuous function defined on $[0, \infty)$. When talking about $V_{p}(t, x)$ defined on $[0, \infty) \times s_{Q}$ it will be understood that in $(3.2)$ the class of functions $\varphi \in A(t, x)$ will have the additional restriction that $|\varphi|<\varrho$.

Lemma 1. - Let $\psi(t)$ be any positive nondecreasing continuous function. Assume $u\left(t, t_{0}, u_{0}\right)$ is a solution of (S) satisfying

$$
\left|\frac{\partial u\left(t, 0, u_{0}\right)}{\partial u_{0}}\right| \leqslant M \sigma(t)
$$


for some $M>0$, where $\sigma(t):[0, \infty) \rightarrow(0, \infty)$ is continuous and such that

$$
\sigma(t) \psi(t) \leqslant N
$$

for some $N>0$ and $t \in[0, \infty)$. Then $W_{y}(t, x)$ is a nonnegative continuous function on $[0, \infty) \times S_{Q}$ and satisfies $(2.1)$ and $(2.2)$.

REMARK. - For the special case $\psi(t) \equiv 1$ and $h(t, u) \equiv 0, W_{\psi}(t, x)$ reduces to the Okamura function $V^{0}(t, x)$ which has been shown in [7] to satisfy (2.1) and (2.2),

Using Lemma 1 we now obtain a generalization of the uniqueness of zero solution of (E) presented in [7].

Theorem 1. - Assume $f(t, 0)=h(t, 0) \equiv 0$. Then if there exists a continuous function $V(t, x)$ defined on $[0, \infty) \times S_{e}$ satisfying $(2.1),(2.2)$, and

$$
V(t, 0)=0 \text { and } V(t, x)>0 \text { for }|x| \neq 0
$$

the zero solution of $(\mathrm{E})$ is unique.

Conversely, assume the zero solution of $(\mathbf{E})$ is unique in the future. Let $h(t, u)$ be any function satisfying $h(t, 0) \equiv 0$ such that the solutions of (S) satisfy (3.3) and

$$
u\left(t, 0, u_{0}\right) \geqslant \lambda(t) \chi\left(u_{0}\right)
$$

where $\chi:[0, \infty) \rightarrow[0, \infty)$ is continuous, $\chi(0)=0$, and $\chi\left(u_{0}\right)>0$ for $u_{0} \neq 0$, and $\lambda(t):[0, \infty) \rightarrow(0, \infty)$ is continuous. For each nondecreasing positive function $\psi(t)$ satisfying (3.4) there exists a Liapunow function $W_{\psi}(t, x)$ defined on $[0, \infty) \times S_{e}$ satisfying (2.1), (2.2), and (3.5).

For the case $h(t, u) \equiv 0$ and $\psi \equiv 1$ we get as a corollary the following resltu of Yoshtzawa [7].

Corollary 1. - Assume $f(t, 0) \equiv 0$. Then the zero solution of $(\mathrm{E})$ is unique in the future if and only if there exists a continuous Liapunov function satisfying (2.1), (3.5) and $\dot{V}_{E}(t, x) \leqslant 0$.

Our next result generalizes the result of OHow and YoRkE [2] concerning integral stability.

Theoreir 2. - Assume $f(t, 0) \equiv h(t, 0) \equiv 0$ and that the zero solution of (S) is integrally stable. If there exists a continuous Liapunor function $V(t, x)$ defined on $[0, \infty) \times S_{e}$ satisfying $(2.1),(2.2)$ and

$$
a(|x|) \leqslant V(t, x) \leqslant b\left(\mid x_{i}^{\prime}\right),
$$


where $a:[0, \varrho) \rightarrow[0, \infty)$ is continuous and strictly increasing and $b:[0, \varrho) \rightarrow[0, \infty)$ is continuous with $b(0)=0$, then the zero solution of $(\mathrm{E})$ is integrally stable. Conversely, assume the zero solution of $(\mathrm{E})$ is integrally stable. Let $h(t, u)$ be any function such that the solutions of (S) satisfy (3.3) and (3.6) for $\lambda(t) \geqslant C>0$. Then sor each nondecreasing positive function $\psi(t)$ satisfying (3.4) there exists a continuous Liapunov function $W_{\psi}(t, x)$ defined on $[0, \infty) \times S_{\varrho}$, satisfying $(2.1),(2.2)$, and (3.7).

REMARK. - If we again let $h(t, u) \equiv 0$ and $\psi \equiv 1$ then since the zero solution of $\dot{u}=0$ is integrally stable we obtain the following result of CHow and Yorke [2] as a corollary of Theorem 2.

CoroluaRY 2. - The zero solution of $(\mathbf{E})$ is integrally stable if and only if there exists a continuous Liapunov function defined in $[0, \infty) \times S_{\varrho}$ satisfying $(2.1),(3.7)$, and $\dot{V}_{g}(t, x) \leqslant 0$.

The following theorem generalizes a result of OHOW and YoRKE [2] concerning the integrally asymptotic stability of the zero solution of (E).

THeOREM 3. - Assume $f(t, 0)=h(t, 0) \equiv 0$ and that the zero solution of (S) is integrally asymptotically stable. Then if there exists a Liapunov function defined on $[0, \infty) \times S_{e}$ satisfying $(2.1),(2.2)$, and $(3.7)$, where $a(\cdot)$ and $b(\cdot)$ satisfy the same conditions as in Theorem 2 , then the zero solution of $(\mathrm{E})$ is integrally asymptotically stable. Conversely, assume the zero solution of $(\mathrm{E})$ is integrally asymptotically stable. Let $h(t, u)$ be any function such that the solutions of (S) satisfy (3.3), where $\sigma(t) \rightarrow 0$ as $t \rightarrow \infty$, and (3.6) with $\lambda(t) \equiv \sigma(t)$, and $\chi(u)=u$. Then for each nondecreasing positive function $\psi(t)$ satisfying (3.4) and

$$
\lim _{t \rightarrow \infty} \inf \sigma(t+r) \psi(t) \geqslant \mu(r),
$$

for each $r>0$, where $\mu(r)>0$, there exists a continuous Liapunov function $W_{\psi}(t, x)$ defined on $[0, \infty) \times \mathbb{S}_{e}$ satisfying (2.1), (2.2) and (3.7).

REMARK. - If we let $h(t, u) \equiv-u, \sigma(t)=e^{-t}, \psi(t)=e^{t}$ then since the zero solution of $\dot{u}=-u$ is integrally asymptotically stable the conditions of Theorem 3 hold and we obtain the following result of OHOW and YoRke [2] as a corollary of Theorem 3.

CoRollaRY 3. - The zero solution of $(\mathrm{E})$ is integrally asymptotically stable if and only if there exists a continuous Liapunov function defined on $[0, \infty) \times S_{e}$ satisfying (2.1), $(3.7)$, and $\dot{V}_{B}(t, x) \leqslant-V$.

The next two results generalize the results on integral boundedness and extendability obtained in [1]. In these results we of course do not assume that $x(t) \equiv 0$ is solution of $(\mathrm{E})$ and do construct our Liapunov function on $[0, \infty) \times R^{a}$.

Theorem 4. - Assume solutions of (S) are integrally bounded. Moreover, assume there exists a continuous Liapunov function defined on $[0, \infty) \times R^{a}$, satisfying (2.1), 
(2.2), and (3.7) where now $a(r) \rightarrow \infty$ as $r \rightarrow \infty$ monotonically. Then solutions of (E) are integrally bounded. Oonversely, assume solutions of (E) are integrally bounded. Let $h(t, u)$ be any function such that $u(t, 0,0)$ is bounded in the future, and assume solutions of (S) satisfy (3.3) and (3.6) for $\lambda(t) \geqslant C>0$. Then for each nondecreasing positive function $\psi(t)$ satisfyng (3.4) there exists a continuous Liapunov function $W_{\varphi}(t, x)$ defined on $[0, \infty) \times R^{d}$ satisfying $(2.1),(2.2)$, and $(3.7)$ where once again $a(r) \rightarrow \infty$ as $r \rightarrow \infty$ monotonically.

Remark. - Again letting $h(t, u) \equiv 0$ and $\psi \equiv 1$ and noting that the solutions of $\dot{u}=0$ are integrally bounded we obtain the following result of CHow and one of the authors [1] as a corollary of Theorem 4 :

Corollarr 4. - The solutions of (E) are integrally bounded if and only if there exists a continuous Liapunov function $V(t, x)$ defined on $[0, \infty) \times R^{d}$ satisfying (2.1), (3.7) (with $a(r) \rightarrow \infty$ as $r \rightarrow \infty$ monotonically) and $\dot{V}_{E}(r, x) \leqslant 0$.

A theorem anaogous to Theorem 4 may be stated for the case of integral extendability. The only change is that instead of satisfying $(3.7), V(t, x)$ satisfies $V(t, x) \rightarrow \infty$ as $|x| \rightarrow \infty$, uniformly for $t$ in compact sets of $[0, \infty)$. We will omit the details.

In the next theorem we will obtain sufficient conditions for the integral boundedness and integral ultimate boundedness of the solutions of $(E)$ in terms of a scalar differential equation whose solutions are uniformly bounded and uniformly ultimately bounded.

THEorexr 5. - Assume there exists a continuous Liapunov function defined on $[0, \infty) \times R^{a}$, satisfying (2.1), (2.2), and (3.7), where $a(r) \rightarrow \infty$ as $r \rightarrow \infty$ monotonically. Let the function $h(t, u)$ be nonincreasing in $u$ for each $t$. Then the uniform boundedness of solutions of (S) implies the integral boundedness of solutions of (E) and the uniform ultimate boundedness of solutions of (S) implies the integral ultimate boundedness of solutions of (E).

REMARK. - We have not obtained a converse result in the case of integral ultimate boundedness as we did for the previous cases. This remains an open question.

\section{4. - Proofs.}

Proof of Lemra 1. - Let $\psi$ be any positive nondecreasing continuous function and let $W_{\psi}$ and $V_{\psi}$ be defined as in (3.1) and (3.2). First we show $W_{\psi}$ satisfies (2.1). From (3.3)

$$
\left|W_{\psi}(t, x)-W_{\psi}(t, y)\right| \leqslant M \sigma(t)\left|V_{y}(t, x)-V_{\psi}(t, y)\right|,
$$

In view of (3.4) it is sufficient to prove

$$
\left|V_{\psi}(t, x)-V_{\psi}(t, y)\right| \leqslant \psi(t)|x-y| .
$$


For any $\varepsilon>0$ pick $\varphi \in A(t, x)$ such that

$$
\int_{0}^{t} \psi(s) \mid \dot{\varphi}(s)-f\left(s, \varphi(s)\left(\mid d s \leqslant V_{\eta}(t, x)+\varepsilon\right.\right.
$$

For each $h>0$ define

$$
\varphi_{h}(s)=\left\{\begin{array}{l}
\varphi(t-h)+\frac{y-\varphi(t-h)}{h}(s-(t-h)), \quad s \in[t-h, t] . \\
\varphi(s), \quad s \in[0, t-h]
\end{array}\right.
$$

Since $\varphi_{h} \in A(t, y)$

$$
\begin{aligned}
V_{\psi}(t, y) \leqslant & \int_{0}^{t} \psi(s)\left|\dot{\varphi}_{h}(s)-f\left(s, \varphi_{h}(s)\right)\right| d s \\
& =\int_{0}^{t-h} \psi(s)|\dot{\varphi}(s)-f(s, \varphi(s))| d s+\int_{t-h}^{t} \psi(s)\left|\dot{\varphi}_{h}(s)-f\left(s, \varphi_{h}(s)\right)\right| d s \\
& \leqslant \int_{0}^{t-h} \psi(s)|\dot{\varphi}(s)-f(s, \varphi(s))| d s+\psi(t)\left|\int_{t-h}^{t} \dot{\varphi}_{h}(s)\right| d s+\psi(t) \int_{i-h}^{t}\left|f\left(s, \varphi_{h}(s)\right)\right| d s .
\end{aligned}
$$

There exists an $M>0$ such that $\left|f\left(s, \varphi_{h}(s)\right)\right| \leqslant M$ for $s \in[t-h, t]$ and $h>0$. Hence,

$$
V_{\varphi}(t, y) \leqslant \int_{0}^{t-h} \psi(s)|\dot{\varphi}(s)-f(s, \varphi(s))| d s+\psi(t)|y-\varphi(t-h)|+M h \psi(t)
$$

since $\varphi_{h}(t)=y$ for all $h$. Letting $h \rightarrow 0(4.3)$ becomes

$$
V_{\psi}(t, y) \leqslant \int_{0}^{t} \psi(s)|\dot{\varphi}(s)-f(s, \varphi(s))| d s+\psi(t)|y-x| \leqslant V_{\psi}(t, x)+\varepsilon+\psi(t)|y-x| .
$$

Since this is true for every $\varepsilon>0$

$$
V_{\psi}(t, y) \leqslant V_{\psi}(t, x)+\psi(t)|y-x| \text {. }
$$

Interchanging the roles of $x$ and $y$ we obtain (4.2). then

We now show $W_{\psi}(t, x)$ satisfies the Lipschitz condition

$$
\left|W_{\psi}(t, x)-W_{\psi}(s, x)\right| \leqslant N|t-s|,
$$


where $N$ depends on $s, t$, and $x$. Then (4.4) and (2.1) will yield the continuity of $W_{\psi}$ in $[0, \infty) \times R^{d}$. Now

$$
\begin{aligned}
\left|W_{\psi}(t, x)-W_{\psi}(s, x)\right| & =\left|u\left(t, 0, V_{\psi}(t, x)\right)-u\left(s, 0, V_{\psi}(s, x)\right)\right| \\
& \leqslant\left|u\left(t, 0, V_{\psi}(t, x)\right)-u\left(s, 0, V_{\psi}(t, x)\right)\right|+ \\
& +\left|u\left(s, 0, V_{\psi}(t, x)\right)-u\left(s, 0, V_{\psi}(s, x)\right)\right|
\end{aligned}
$$

Since $\dot{u}(t)$ is continuous there exists $N_{1}$ depending on $t, s$, and $V_{y}(t, x)$ such that

$$
\left|u\left(t, 0, V_{\psi}(t, x)\right)-u\left(s, 0, V_{\psi}(t, x)\right)\right| \leqslant N_{1}|t-s| \text {. }
$$

Moreover, from (3.3)

$$
\left|u\left(s, 0, V_{\psi}(t, x)\right)-u\left(s, 0, V_{\psi}(s, x)\right)\right| \leqslant M \sigma(s)\left|V_{\psi}(t, x)-V_{\psi}(s, x)\right| .
$$

It remains to show that

$$
\left|V_{\psi}(t, x)-V_{\psi}(s, x)\right| \leqslant N_{2}|t-s|
$$

where $N_{2}$ depends on $t, s$, and $x$; for then with (4.5), (4.6) and (4.7) we would arrive at (4.4).

Assume $t>s$. For any $\varepsilon>0$ choose $\varphi \in A(s, x)$ such that

$$
\int_{0}^{s} \psi(r)|\dot{\varphi}(r)-f(r, \varphi(r))| d r \leqslant V_{\psi}(s, x)+\varepsilon
$$

Define

$$
\varphi_{1}(r)= \begin{cases}\varphi(r), & r \in[0, s] \\ x, & r \in[s, t]\end{cases}
$$

Then

$$
V_{\psi}(t, x) \leqslant \int_{0}^{s} \psi(r)\left|\dot{\varphi}_{1}(r)-f\left(r, \varphi_{1}(r)\right)\right| d r=\int_{0}^{t} \psi(r)|\dot{\varphi}(r)-f(s, \varphi(r))| d r+\int_{s}^{t} \psi(r)|f(r, x)| d r,
$$

thus yielding

$$
V_{\psi}(t, x) \leqslant V_{\psi}(s, x)+\varepsilon+\psi(t) M(t-s),
$$

where $|f(r, x)| \leqslant M$ for $r \in[s, t]$. Since $\varepsilon$ is arbitrary

$$
V_{\psi}(t, x) \leqslant V_{\psi}(s, x)+\psi(t) M(t-s) .
$$

7 - Annali di Matematica 
Pick any $\varphi \in A(t, x)$. Then

$$
\begin{aligned}
\int_{0}^{t} \psi(r)|\dot{\varphi}(r)-f(r, \varphi(r))| d r & =\int_{0}^{s} \psi(r)|\dot{\varphi}(r)-f(r, \varphi(r))| d r+\int_{s}^{t} \psi(r)|\dot{\varphi}(r)-f(r, \varphi(r))| d r \\
& \geqslant \int_{0}^{s} \psi(r)|\dot{\varphi}(r)-f(r, \varphi(r))| d r+\int_{s}^{t} \psi(r)|\dot{\varphi}(r)| d r-\int_{s}^{t} \psi(r)|f(r, \varphi(r))| d r \\
& \geqslant \nabla_{\psi}(s, \varphi(s))+\psi(s)|x-\varphi(s)|-\psi(t) M_{1}(t-s)
\end{aligned}
$$

where $M_{1}=\sup _{\substack{r \in 0, t] \\ y \in s_{p}}}|f(r, y)|$. Since this inequality holds for all $\varphi \in A(t, x)$ we have

$$
V_{\psi}(t, x) \geqslant V(s, \varphi(s))+\psi(s)|x-\varphi(s)|-\psi(t) M_{1}(t-s) .
$$

From (4.2)

$$
V_{\psi}(s, \varphi(s))+\psi(s)|x-\varphi(s)| \geqslant V_{\psi}(s, x)
$$

and thus

$$
V_{\psi}(t, x) \geqslant V_{\psi}(s, x)-\psi(t) M_{1}(t-s)
$$

Hence (4.9) and (4.10) yield

$$
\left|V_{\psi}(t, x)-V_{\psi}(s, x)\right| \leqslant \psi(t) M_{2}|t-s|
$$

where $M_{2}=\max \left(M, M_{1}\right)$ and for $t>s$. For $s>t$ a similar type of inequality is obtained and, therefore, (4.8) is satisfied.

It remains to show (2.2) is satisfied. For fixed $t$ let $x(s)$ be a solution of $(\mathrm{E})$ satisfying $x(t)=x$. For each $\varepsilon>0$ pick $\varphi \in A(t, x)$ such that

$$
\int_{0}^{t} \psi(s)|\dot{\varphi}(s)-f(s, \varphi(s))| d s \leqslant V_{\psi}(t, x)+\varepsilon .
$$

Define

$$
\varphi_{k}(s)= \begin{cases}\varphi,(s) & s \in[\mathbf{0}, t] \\ x(s), & s \in[t, t+h] .\end{cases}
$$

Since $u\left(t, 0, r_{0}\right)$ is a solution of $(\mathbf{S})$ and solutions are uniquely determined by initial 
conditions then $\partial u\left(t, 0, r_{0}\right) / \partial r_{0} \geqslant 0$. Thus

$$
\begin{aligned}
W_{\psi}(t+h, x(t+h)) & =u\left(t+h, 0, \nabla_{\psi}(t+h, x(t+h))\right) \\
& \leqslant u\left(t+h, 0, \int_{0}^{t+h} \psi(s)\left|\dot{\varphi}_{h}(s)-f\left(s, \varphi_{h}(s)\right)\right| d s\right) \\
& =u\left(t+h, 0, \int_{0}^{t} \psi(s)|\dot{\varphi}(s)-f(s, \varphi(s))| d s+\left|\int_{t}^{t+h} \dot{x}(s)-f(s, x(s))\right| d s\right) \\
& =u\left(t+h, 0, \int_{0}^{t} \psi(s)|\dot{\varphi}(s)-f(s, \varphi(s))| d s\right) \leqslant u\left(t+h, 0, V_{\psi}(t, x)+\varepsilon\right) .
\end{aligned}
$$

Since $\varepsilon$ is arbitrary

$$
W_{\psi}(t+h, \infty(t+h)) \leqslant u\left(t+h, 0, \nabla_{\psi}(t, x)\right) .
$$

Therefore

$$
W_{\psi}(t+h . x(t+h))-W_{\psi}(t, x) \leqslant u\left(t+h, 0, V_{\psi}(t, x)\right)-u\left(t, 0, V_{\psi}(t, x)\right)
$$

Dividing (4.11) by $h>0$ and letting $h \rightarrow 0^{+}$we get

$$
\dot{W}_{\psi}(t, x) \leqslant \dot{u}\left(t, 0, V_{\psi}(t, x)\right)=h\left(t, u\left(t, 0, V_{\psi}(t, x)\right)\right)=h\left(t, W_{\psi}(t, x)\right),
$$

thus yielding (2.2) and concluding the proof of Lemma 1.

Proof of Theorem 1. - For any point $\left(t_{0}, 0\right), V\left(t_{0}, 0\right)=0$ and from $(2.1)$ and $(2.2)$.

$$
0 \leqslant V\left(t, x\left(t, t_{0}, 0\right)\right) \leqslant u\left(t, t_{0}, V\left(t_{0}, 0\right)\right)=0
$$

for $t=t_{0}$. Hence $V\left(t, x\left(t, t_{0}, 0\right)\right)=0$ for $t=t_{0}$; this implies $x\left(t, t_{0}, 0\right)=0$.

Conversely, define $W_{\psi}(t, x)$ as in (3.1). From Lemma $1 W_{\psi}(t, x)$ is continuous in $[0, \infty) \times S_{\varrho}$ and satisfies (2.1) and (2.2). It remains to show $W_{\psi}(t, x)$ satisfies (3.5). From (3.6)

$$
W_{\psi}(t, x)=u\left(t, 0, V_{\psi}(t, x)\right) \geqslant \lambda(t) \chi\left(V_{\psi}(t, x)\right)
$$

It has been proved by YosHIZAWA [7] that the uniqueness of the zero solution of (E) implies $V_{\psi}(t, 0)=0$ and $V_{\psi}(t, x)>0$ when $|x|>0$ for $\psi \equiv 1$. A similar argument holds for any now decreasing positive $\psi(t)$. From the conditions on $\chi(\cdot),(3.5)$ follows.

Proof of THeorem 2. - The first half of Theorem 2 (sufficiency) has essentially been proved by LakshmmaNTHAM and LeELA [4] and we omit the detai]s. 
Conversely, define $W_{\psi}(t, x)$ as in the previous theorem. From Lemma $1 W_{\psi}(t, x)$ satisfies (2.1) and (2.2). It thus remains to prove (3.7). From (2.1), and the fact that $W_{\psi}(t, 0)=0$, we obtain $W_{\psi}(t, x) \leqslant K|x|$. Moreover, from (3.6), and the lower bounds on $\lambda(\cdot)$ and $\psi(\cdot)$

$$
W_{\psi}(t, x)=u\left(t, 0, V_{\psi}(t, x)\right) \geqslant C V_{\psi}(t, x) \geqslant C_{\psi}(0) V^{0}(t, x)
$$

where $V^{0}(t, x)$ is defined in (3.0). Tt has been proved by CHOw and YoRke [2] that $V^{0}(t, x)$ satisfies the left hand inequality of $(3.7)$. Thus $W_{y}(t, x)$ satisfies the left hand inequality of (3.7). The proof of Theorem 2 is complete.

Proof of Theonem 3. - The sufficiency follows from the proof given by LaksirMIKaNTHAM and LeELa [4].

Conversely, define $W_{\psi}(t, x)$ as in Theorem 1 , with $\varrho=\delta_{0}$, where $\delta_{0}$ corresponds to $\delta_{0}$ in Definition 2. From Lemma $1 W_{\psi}(t, x)$ satisfies (2.1) and (2.2). From (2.1), and the fact that $W_{\psi}(t, 0)=0$, we obtain $W_{\psi}(t, x) \leqslant K|x|$. It thus remains to prove the left hand inequality of (3.7). From (3.6)

$$
W_{\psi}(t, x)=u\left(t, 0, V_{\psi}(t, x)\right) \geqslant \sigma(t) V_{\psi}(t, x)=\inf _{q \in A(t, x)} \int_{0}^{t} \sigma(t) \psi(s)|\dot{\varphi}(s)-f(s, \varphi(s))| d s .
$$

It is sufficient to show $\sigma(t) V_{\psi}(t, x)$ satisfies the left hand inequality of (3.7). From the uniqueness of the zero solution of $(\mathrm{E})$ it follows from Theorem 1 that $\sigma(t) V_{\psi}(t, x)>0,|x| \neq 0$. Thus if $\sigma(t) V_{\psi}(t, x)$ does not satisfy the left hand linequality of (3.7), then there exists an $\varepsilon>0$, sequences $\left\{x_{n}\right\}$ and $\left\{t_{n}\right\}$ such that $t_{n} \rightarrow \infty$ and $\varepsilon \leqslant\left|x_{n}\right|$ such that $\sigma\left(t_{n}\right) V_{\psi}\left(t_{n}, x_{n}\right) \rightarrow 0$ as $n \rightarrow \infty$ Let $T(\varepsilon)$ and $\eta(\varepsilon)$ be the numbers corresponding to those in Definition 2.

Choose $N_{1}$ sufficiently large so that for $n>N_{1}, t_{n}>T+1$ and

$$
\sigma\left(t_{n}\right) \nabla_{\psi}\left(t_{n}, x_{n}\right)<\frac{\mu(T+1)}{2} \eta
$$

Pick $\bar{t}$ so large that for $t \geqslant \bar{t}$

$$
\sigma(t+T+1) \psi(t) \geqslant \frac{\mu(T+1)}{2} .
$$

Select $N>N_{1}$ such that $t_{N}-(T+1) \geqslant \bar{t}$ and define $t_{0}=t_{M}-(T+1)$. Then from (4.12), choose $\varphi_{N} \in A(t, x),\left|\varphi_{N}\right|<\delta_{0}$, such that

$$
\int_{0}^{t_{N}} \sigma\left(t_{N}\right) \psi(s)\left|\dot{\varphi}_{N}(s)-f\left(s, \varphi_{N}(s)\right)\right| d s<\frac{\eta \mu(T+1)}{2}
$$


StEphen R. Berneeld - V. LaKshmKantham: Differential inequalities, ete. 101

$$
\begin{aligned}
& \Rightarrow \int_{t_{0}}^{t_{N}} \sigma\left(t_{N}\right) \psi(s)\left|\dot{\varphi}_{N}(s)-f\left(s, \varphi_{N}(s)\right)\right| d s<\frac{\eta \mu(T+1)}{2}, \\
& \Rightarrow \sigma\left(t_{N}\right) \psi\left(t_{0}\right) \int_{t_{0}}^{t_{N}}\left|\dot{\varphi}_{N}(s)-f\left(s, \varphi_{N}(s)\right)\right| d s<\frac{\eta \mu(T+1)}{2} .
\end{aligned}
$$

From (4.13)

Define

$$
\begin{aligned}
& \frac{\mu(T+1)}{2} \int_{t_{0}}^{t_{N}}\left|\dot{\varphi}_{N}(s)-f\left(s, \varphi_{N}(s)\right)\right| d s<\frac{\eta \mu(T+1)}{2}, \\
\Rightarrow & \int_{t_{\theta}}^{t_{N}}\left|\dot{\varphi}_{N}(s)-f\left(s, \varphi_{N}(s)\right)\right| d s<\eta .
\end{aligned}
$$

$$
p_{N}(t)= \begin{cases}0 & \text { for } t \in\left[0, t_{0}\right) \\ \dot{\psi}_{N}(t)-f\left(t, \psi_{N}(t)\right) & \text { for } t \in\left[t_{0}, t_{N}\right] \\ 0 & \text { for } t \in\left[t_{N}, \infty\right)\end{cases}
$$

Let $x_{N}(t)$ be a solution of

$$
\dot{x}=f(t, x)+p_{N}(t)
$$

such that $x_{N}(t)=\psi_{N}(t)$ for $t \in\left[t_{0}, t_{N}\right]$. Since $\int_{0}^{\infty}\left|p_{N}(t)\right| d t<\eta$ for sufficiently large $N$ then from the definition of integral asymptotic stability

$$
\left|x_{N}\left(t, t_{0}, \psi_{N}\left(t_{0}\right)\right)\right|<\varepsilon
$$

for $t \geqslant t_{0}+T$. Choosing $t=t_{N}$, we have

$$
\left|x_{N}\right|=\left|\psi_{N}\left(t_{N}\right)\right|=\left|x_{N}\left(t_{N}, t_{0}, \psi_{N}\left(t_{0}\right)\right)\right|<\varepsilon,
$$

a contradiction to the assumption $\left|x_{N}\right| \geqslant \varepsilon$. This completes the proof of Theorem 3 .

Proof of Theorem 4. - Assume there exists a Liapunov function $V$ satisfying the conditions of the theorem. Then

$$
\dot{V}_{P}(t, x) \leqslant \dot{V}_{E}(t, x)+K|g(t)| \leqslant h(t, V(t, x))+K|g(t)|
$$

For any $t_{0}, x_{0}$ let $u\left(t_{0}, u_{0}\right)$ satisfy

$$
\dot{u}=h(t, u)+K|g(t)|
$$


where $u_{0}=\nabla\left(t_{0}, x_{0}\right)$. Assuming $\left|x_{0}\right|<\alpha, \int_{0}^{\infty}|g(t)| d t<\eta$, we obtain

$$
u\left(t, t_{0}, u_{0}\right) \leqslant \beta(b(\alpha), K \eta)
$$

since $\left|u_{0}\right|<b(\alpha)$ and solutions of (S) are integrally bounded. From $(4,14),(4.15)$ and $(3.7)$

$$
\begin{aligned}
a\left(\left|x_{p}\left(t, t_{0}, x_{0}\right)\right|\right) & \leqslant V\left(t, x_{p}\left(t, t_{0}, x_{0}\right)\right) \leqslant u\left(t, t_{0}, u_{0}\right) \\
& \leqslant \beta(b(\alpha), K \eta) .
\end{aligned}
$$

Hence

$$
\left|x_{p}\left(t, t_{0}, x_{0}\right)\right| \leqslant a^{-1}(\beta(b(\alpha), K \eta)) \equiv \beta_{1}(\alpha, \eta),
$$

thus proving solutions are integrally bounded.

Conversely, define $W_{\psi}(t, x)$ on $[0, \infty) \times R^{d}$ as in (3.1). Now that the domain of $W_{\psi}(t, x)$ is no longer restricted to $[0, \infty) \times S_{e}$ certain changes have to be made. From the proof of Lemma 1 it follows that once again $W_{y}(t, x)$ satisfies (2.1) and (2.2). In proving the continuity of $W_{\psi}$ it was necessary in Lemma 1 to show that $V_{\psi}(t, x)$ satisfies a Lipschitz condition in $t$ and we used the fact that $|f(s, y)|$ is bounded in $[0, t) \times S_{Q}$ in order to obtain the estimate $(4.10)$. To prove $(4.10)$ in the present case let us consider a sequence $\varphi_{k} \in A(t, x)$ such that

$$
V_{\psi}(t, x)=\lim _{k \rightarrow \infty} \int_{0}^{t} \psi(s)\left|\dot{\varphi}_{k}(s)-f\left(s, \varphi_{k}(s)\right)\right| d s .
$$

It is sufficient to show $\left\{\varphi_{k}(t)\right\}$ is uniformly bounded on $[0, t]$ for then $\left|f\left(s, \varphi_{k}(s)\right)\right|$ will be uniformly bounded on $[0, t]$ for all $k$ and we will thus be able to obtain an estimate similar to $(4.10)$, hence proving the continuity of $W_{\psi}(t, x)$ on $[0, \infty) \times R^{d}$. Define

$$
p_{k}(s)= \begin{cases}\dot{\varphi}_{k}(s)-f\left(s, \varphi_{k}(s)\right), & 0 \leqslant s \leqslant t \\ 0 & t \leqslant s \leqslant \infty\end{cases}
$$

Then from (4.16) $\int_{0}^{\infty} \psi(s)\left|p_{k}(s)\right| d s<K_{1}$ for some $K_{1}>0$ which implies $\int_{0}^{\infty}\left|p_{k i}(s)\right| d s<$ $<K_{1} / \psi(0)$ since $\psi(t) \geqslant \psi(0)>0$. Let $x_{k}(s)$ be a solution of

$$
\dot{x}=f(s, x)+p_{k}(s)
$$

in which $x_{k}(s)=\varphi_{k}(s)$ for $s \in[0, t]$. Since solutions of $(\mathrm{E})$ are intgrally bounded $\left|x_{k}(s)\right| \leqslant \beta\left(0, K_{1} / \psi(0)\right)$ for all $k$ and $s \in[0, \infty)$. Hence $\left\{\varphi_{k}(s)\right\}$ is uniformly bounded on $[0, t]$. 
Ir remains to prove $(3.7)$, From (3.6) and the lower bounds on $\lambda(\cdot)$ and $\psi(\cdot)$

$$
W_{\psi}(t, x)=u\left(t, 0, \nabla_{\psi}(t, x)\right) \geqslant C V_{\psi}(t, x)=C \psi(0) \nabla^{0}(t, x),
$$

where $V^{0}(t, x)$ is defined in (3.0). In [1] it has been proved that $V^{0}(t, x)$ satisfies the left hand inequality in (3.7) where $a(r) \rightarrow \infty$ as $r \rightarrow \infty$ monotonically.

Met $\bar{x}(t)$ be a solution of (E) satisfying $\bar{x}(0)=0$. Since $V_{\psi}(t, \vec{x}(t)) \equiv 0$ for all $t$

$$
W_{y}(t, \bar{x}(t))=u(t, 0,0) .
$$

Since $u(t, 0,0)$ is bounded then $\left|W_{p}(t \bar{x}(t))\right| \leqslant K_{2}$. Hence from $(2.1)$

$$
\left|W_{\psi}(t, x)\right| \leqslant\left|W_{\psi}(t, \bar{x}(t))\right|+K|x-\bar{x}(t)| \text {. }
$$

Since solutions of (E) are integrally bounded there exists $K_{3}>0$ such that $|\bar{x}(t)| \leqslant K_{3}$. Therefore

$$
\left|W_{\psi}(t, x)\right| \leqslant \Pi_{2}+K K_{3}+K|x|
$$

that is $W_{\psi}(t, x)$ satisfies the right hand inequality of (3.7). This completes the proof of Theorem 4 .

Proof of Theorem 5. - Let $x\left(t, t_{0}, x_{0}\right)$ be a solution of $(P)$ with $\left|x_{0}\right| \leqslant \alpha$ for some $\alpha>0$. From (2.1) and (3.7), $V\left(t_{0}, x_{0}\right) \leqslant b(0)+K \alpha$. Define

$$
m(t)=V(t, x(t))-K \int_{0}^{t}|g(s)| d s .
$$

Then

$$
\begin{aligned}
\dot{m}(t) & =\dot{V}_{P}(t, x(t))-K|g(t)| \\
& \leqslant \dot{V}_{E}(t, x(t)) \leqslant h(t, V(t, x(t)))
\end{aligned}
$$

(where $\left.\dot{m}(t) \equiv \lim \sup _{h \rightarrow 0^{+}}(m(t+h)-m(t)) / h\right)$. Since $m(t) \leqslant V(t, x(t))$ and $h(t, u)$ is nonincreasing in $u$ it follows that

$$
\dot{m}(t) \leqslant h(t, m(t))
$$

which implies from the theory of differential inequalities

$$
m(t) \leqslant u\left(t, t_{0}, u_{0}\right)
$$

where $u$ is the solution of (\$) through $\left(t_{0}, u_{0}\right)$ with $u_{0}=m\left(t_{0}\right)$. Since $m\left(t_{0}\right) \leqslant V\left(t_{0}, x_{0}\right) \leqslant$ $\leqslant b(0)+K \alpha$ and solutions of (S) are uniformly bounded

$$
\left|u\left(t, t_{0}, u_{0}\right)\right| \leqslant \beta_{1}(b(0)+K \alpha)
$$


for all $t \geqslant t_{0}$, where $\beta_{1}(r)$ is an increasing function such that $\beta_{1}(r) \rightarrow \infty$ as $r \rightarrow \infty$. Assume now $\int_{0}^{\infty}|g(t)| d t<\eta$. Then from (3.7), (4.17), and (4.18)

$$
a\left(\left|x_{P}\left(t, t_{0}, x_{0}\right)\right|\right) \leqslant V\left(t, x_{P}(t)\right) \leqslant u\left(t, t_{0}, x_{0}\right)+K \int_{t_{0}}^{t}|g(s)| d s \leqslant \beta_{1}(b(0)+K \alpha)+K \eta
$$

hence

$$
\left|x_{p}\left(t, t_{0}, \hat{x}_{0}\right)\right| \leqslant a^{-1}\left(\beta_{1}(b(0)+K \alpha)+K \eta\right)
$$

thus proving solutions of (E) are integrally bounded.

Assume now solutions of (S) are uniformly ultimately bounded. Repeating the proof of the first half of the theorem through (4.17) we obtain the following estimate on $u\left(t, t_{0}, u_{0}\right)$ from the uniform ultimate boundedness of the solutions of (S):

$$
\left|u\left(t, t_{0}, u_{0}\right)\right| \leqslant B
$$
for $t \geqslant t_{0}+T(b(0)+K \alpha)$ where $B$ is a fixed constant. If we assume again $\int_{0}^{\infty}|g(t)| d t<\eta$,
then $(3.17),(4.17)$, and (4.19) imply

$$
a\left(\left|x_{p}\left(t, t_{0}, x_{0}\right)\right|\right) \leqslant V\left(t, x_{p}(t)\right) \leqslant B+K \eta
$$

for $t \geqslant t_{0}+T(b(0)+K \alpha)$; that is

$$
\left|x_{p}\left(t, t_{0}, \infty_{0}\right)\right| \leqslant a^{-1}\left(B+K_{\eta}\right)
$$

for $t \geqslant t_{\mathrm{a}}+T(b(0)+K \alpha)$. Thus solutions of (S) are integrally ultimately bounded proving Theorem 5 .

REMark. - In Theorems (4) and (5) we of course do not require the condition $\delta(0)=0$ in $(3.7)$.

\section{REFERENCES}

[1] S. BERNFELD - S. N. CHOW, The boundedness and extendability of differential systems under integral perturbations, Ann. di Mat. pura e appl., to appear.

[2] S. N. CHOW - J. YORKE, Liapunov theory and perturbation of stable and asymptotically stable systems (to appear).

[3] A. Halanay, Differential Equations, Academic Press, New York, 1966.

[4] V. Lakshmmkantham - S. LeELA, Differential and Integral Inequalities, vol. I, Academic Press, New York, 1969. 
[5] H. OKamuRA, Conditions nécessaire et suffisantes remplie par équations différentielles ordi, naires sans points de Peano, Mem. Call. Sci. Kyoto Imperial Univ., Séries A, 24 (1942). pp. 21-28.

[6] I. VRroc, Integral stability, Czech. Journal of Mathematics, 9 (1959), pp. $71-129$.

[7] T. Yoshizawa, Stability theory by Liapunov's second method, Math. Soc. Japan, Tokyo, 1966. 